\title{
Factores de riesgo para infarto agudo de miocardio y prescripción de medicamentos para prevención secundaria
}

\footnotetext{
Proyecto UCR-CCSS: VI422-A0-I38 Estudios de Utilización de Medicamentos, Escuela de Medicina, Universidad de Costa Rica.

2. Dpto. Farmacoterapia, Caja Costarricense de Seguro Social.
}

Abreviaturas: IAM, infarto agudo de miocardio; AAS, aspirina; IECA, inhibidor de la enzima convertidora de Angiotensina; ARA-2, antagonista del receptor de Angiotensina 2; IMC, índice de masa corporal.

Correspondencia: D. Sáenz, Dpto de Farmacoterapia, piso 12 oficinas centrales CCSS; E-mail: dsaenzc@ccss.sa.cr

ISSN 0001-6002/2005/47/31-35 Acta Médica Costarricense, C2005 Colegio de Médicos y Cirujanos

\begin{abstract}
Desirée Sáenz-Campos ${ }^{1,2}$, Zahira Tinoco-Mora ${ }^{1,2}$, Laura M. Rojas-Mora '
\section{Resumen}

Objetivo: tipificar algunos factores de riesgo coronario presentes en los pacientes que sobreviven a un primer infarto agudo de miocardio (IAM) y describir el tratamiento farmacológico prescrito para profilaxis secundaria al egreso hospitalario.

Métodos: estudio observacional, transversal y descriptivo, con información extraída del expediente clínico de 50 pacientes atendidos en 3 hospitales nacionales.

Resultados: 42 varones y 8 mujeres, edad $63 \pm 15$ años, peso $67 \pm 12 \mathrm{~kg}$, Índice de Masa Corporal $=26.1 \pm 2.1 \mathrm{~kg} / \mathrm{m}^{2}, 38 \%$ hipertensos, $22 \%$ diabéticos $(\mathrm{n}=8$ pacientes con diabetes + hipertensión) y $34 \%$ fumadores. Colesterol total $191 \pm 45.7 \mathrm{mg} / \mathrm{dL}$, colesterol-LDL $112 \pm 33.8$ $\mathrm{mg} / \mathrm{dL}$ y colesterol-HDL $39.4 \pm 8.26 \mathrm{mg} / \mathrm{dL}$. Todos egresaron con medicamentos: $92 \%$ con antitrombóticos (predominó aspirina), 92\% con estatinas, 78\% con betabloqueador (atenolol o carvedilol), $70 \%$ con enalapril o losartán y $48 \%$ con nitratos.

Conclusiones: este estudio piloto mostró que la enfermedad coronaria tiende a manifestarse desde la etapa de adulto joven, persiste en su mayor afectación a los varones y parecen prevalecer los factores de riesgo coronario modificables. Los antitrombóticos, beta-bloqueadores y las estatinas más frecuentemente prescritos.

Descriptores: infarto agudo de miocardio, prevención secundaria, colesterol, aspirina, atenolol, enalapril.

Recibido: 30 de julio de 2004

Aceptado: 7 de diciembre de 2004

Los pacientes que han sufrido un infarto agudo de miocardio poseen un alto riesgo de nuevos eventos cardiovasculares y de muerte, la intervención es por tanto una prioridad en procura de una prevención secundaria de tales eventos. La intervención está definida en 2 sentidos, primero la aplicación de medidas no farmacológicas para adoptar un estilo de vida más saludable y el apoyo con programas de rehabilitación cardiaca; y segundo, la intervención farmacológica ${ }^{1,2}$.

Los cambios en el estilo de vida pueden tener un efecto significativo en la prevención secundaria. Primordialmente, se recomienda suspender el consumo del tabaco, realizar ejercicio físico de forma regular y una dieta balanceada sobre la base de vegetales. El consumo de grasas deberá ser reducido, de tal manera que no contribuya a la hiperlipidemia ${ }^{1}$. En este contexto, la consejería y la educación al paciente, su familia y en general, a la población, en procura de la aplicación de medidas no farmacológicas, es fundamental. 
Por otra parte, como intervención farmacológica de forma consensual se acepta que, si no hay contraindicaciones, todos los pacientes que han sufrido un infarto deben recibir ácido acetilsalicílico (aspirina, AAS) ${ }^{1-4}$. Además, es procedente evaluar la administración de un bloqueador beta-adrenérgico ${ }^{1,2,5}$ y un inhibidor de la enzima convertidora de angiotensina (IECA) ${ }^{1,2,6}$. También, se considera que las estatinas ${ }^{7}$ son útiles para la profilaxis secundaria en pacientes que cursan con dislipidemia ${ }^{1,3}$.

En el ámbito de la Seguridad Social y bajo los principios del uso racional de medicamentos, estos fármacos constituyen un recurso terapéutico oportunamente disponible para beneficiar las personas atendidas, de modo que la utilización real de los medicamentos estaría condicionada por la prescripción médica. En consecuencia, el presente trabajo informa sobre un estudio piloto cuyos objetivos fueron 1 . tipificar algunos factores de riesgo coronario presentes en los pacientes sobrevivientes a un primer infarto agudo de miocardio (IAM) y 2, describir el tratamiento farmacológico prescrito para profilaxis secundaria al egreso hospitalario.

\section{Metodología}

Estudio observacional, descriptivo, transversal, cuyo protocolo original corresponde a un estudio multicéntrico desarrollado en países de América Latina. La participación de Costa Rica en una fase inicial del estudio piloto reportó información preliminar sobre 37 casos.

El protocolo fue aprobado por el Comité Ético Científico Institucional de la Caja Costarricense de Seguro Social; se respetaron los principios éticos inherentes al proceso de investigación con información proveniente de expedientes clínicos, con especial énfasis en la confidencialidad.

La muestra se seleccionó aleatoriamente entre los pacientes egresados vivos con diagnóstico de IAM, como un primer evento al momento del ingreso en un hospital general; se excluyó la información de pacientes que ingresaron por otra causa y presentaron un evento intrahospitalario, así como los que sufrieron un reinfarto durante la hospitalización.

Se recogió información de los primeros 50 pacientes: 8 pacientes del Hospital México, 39 del San Juan de Dios y 3 pacientes del Dr. Calderón Guardia; la misma se obtuvo durante marzo-setiembre de 2003. Toda la información fue compilada por médicos generales.

Se utilizó un formulario estandarizado para recoger los datos del expediente clínico tras el egreso hospitalario; se tomo la edad, el sexo, la talla, peso, las cifras de colesterol total, el colesterol-LDL y el colesterol-HDL durante el internamiento, los antecedentes personales de hipertensión arterial, diabetes, tabaquismo, consumo adicional de sal y realización de ejercicio físico; así como el antecedente materno y paterno de IAM antes de los 65 años. Además, a cada paciente se le calculó el índice de masa corporal $\left(\mathrm{IMC}=\text { peso/altura }{ }^{2}\right)^{8}$. Otros antecedentes personales tomados de la historia clínica fueron: asma bronquial, enfermedad pulmonar obstructiva crónica, bloqueo atrioventricular (AV), hemorragia cerebral, insuficiencia hepática, estenosis de arteria renal, úlcera péptica, hemorragia gastrointestinal, insuficiencia cardiaca, enfermedad arterial periférica, patología tiroidea, enfermedad hemorrágica, angor, hipersensibilidad a aspirina, edema angioneurótico por IECA y embarazo.

El contenido de los formularios fue digitado en una base de datos estandarizada, se procesó la información y se procedió con un análisis mediante la aplicación de estadística descriptiva.

\section{Resultados}

Se incluyeron 50 personas (42 hombres y 8 mujeres) con edad de $63 \pm 15$ años (rango $37-84$ años), peso $=67 \pm$ $12 \mathrm{~kg}$ (rango $47-88 \mathrm{~kg}$ ) e IMC de $26.1 \pm 2.1 \mathrm{~kg} / \mathrm{m}^{2}$ (rango $22.7-29.4 \mathrm{~kg} / \mathrm{m} 2$ ). El peso y de la talla se obtuvieron en el expediente clínico de 27 (54\%) y 12 (24\%) pacientes, respectivamente.

Los diversos antecedentes mórbidos y factores de riesgo se muestran en el cuadro 1 . Se los 11 pacientes diabéticos identificados, 8 también eran hipertensos. Solo 2 pacientes $(4 \%)$ tenían antecedente paterno de cardiopatía isquémica y ninguno lo refirió de la madre. Se registró igual proporción de fumadores y exfumadores, éstos lo habían suspendido desde hacía unos 3 meses hasta 40 años antes del ingreso. Además, se pudo documentar que 4 pacientes habían tenido hemorragia cerebral, 2 con tiroidectomía previa, 1 con alergia a la aspirina y otro con antecedente de hemorragia gastrointestinal e insuficiencia cardiaca.

Durante la hospitalización, la cuantificación del colesterol y sus fracciones plasmáticas se hizo solo en 24 pacientes $(48 \%)$ y en ningún caso el colesterol total superó los $313 \mathrm{mg} / \mathrm{dL}$ (cuadro 2).

En el curso de su evolución intrahospitalaria, 7 pacientes $(14 \%)$ cursaron con angina severa. También, 3 pacientes $(6 \%)$ mostraron bradicardia sinusal, $2(4 \%)$ presentaron flutter, un paciente cursó con bradicardia + angina, otro con bradicardia + flutter + bloqueo AV grado I, otro más con bloqueo AV grado II y otro mostró bloqueo de III grado, edema agudo de pulmón, trombo intraventricular y angor persistente. 


\begin{tabular}{|c|c|c|c|c|c|c|c|c|c|}
\hline \multicolumn{10}{|c|}{$\begin{array}{l}\text { Cuadro 1: Antecedentes mórbidos y factores de riesgo cardiovascular } \\
\text { de } 50 \text { pacientes con un primer IAM egresados vivos. }\end{array}$} \\
\hline factor & si & $\%$ & no & $\%$ & no sabe & $\%$ & sin dato & $\%$ & total \\
\hline Hipertensión & 19 & 38 & 31 & 62 & - & 0 & - & 0 & 50 \\
\hline Diabetes & $11^{*}$ & 22 & 38 & 76 & - & 0 & 1 & 2 & 50 \\
\hline Padre con IAM $<65$ años & 2 & 4 & 37 & 74 & 8 & 16 & 3 & 6 & 50 \\
\hline Madre con IAM < 65 años & 0 & 0 & 41 & 82 & 6 & 12 & 3 & 6 & 50 \\
\hline Fumador & 17 & 34 & $32^{* *}$ & 64 & - & 0 & 1 & 2 & 50 \\
\hline Ingesta adicional de sal & 16 & 32 & - & 0 & - & 0 & 34 & 68 & 50 \\
\hline No ejercicio regular & 11 & 22 & 8 & 16 & - & 0 & 31 & 64 & 50 \\
\hline Otras enfermedades & 8 & 16 & 34 & 68 & - & 0 & 8 & 16 & \\
\hline
\end{tabular}

\begin{tabular}{|c|c|c|}
\hline \multicolumn{3}{|c|}{$\begin{array}{c}\text { Cuadro 2: Perfil lipídico documentado } \\
\text { en } 24 \text { de los } 50 \text { pacientes con un primer IAM } \\
\text { egresados vivos. }\end{array}$} \\
\hline Colesterol & Media \pm DE & rango \\
\hline Total & $191 \pm 45.7 \mathrm{mg} / \mathrm{dL}$ & $112-313 \mathrm{mg} / \mathrm{dL}$ \\
\hline $\begin{array}{l}\text { Fracción LDL } \\
\text { (baja densidad) }\end{array}$ & $112 \pm 33.8 \mathrm{mg} / \mathrm{dL}$ & $40-154 \mathrm{mg} / \mathrm{dL}$ \\
\hline $\begin{array}{l}\text { Fracción HDL } \\
\text { (alta densidad) }\end{array}$ & $39.4 \pm 8.26 \mathrm{mg} / \mathrm{dL}$ & $24-59 \mathrm{mg} / \mathrm{dL}$ \\
\hline
\end{tabular}

Todas las personas egresaron con tratamiento farmacológico en procura del efecto cardiovascular, predominó la prescripción de 4-5 fármacos en el $64 \%$ de los pacientes (figura 1).

La figura 2 muestra los medicamentos prescritos con mayor frecuencia, los antitrombóticos (92\%), las estatinas (92\%), y los beta-bloqueadores (78\%).

Respecto a los antitrombóticos, la prescripción más frecuente fue de aspirina como agente único o de la combinación aspirina + ticlopidina o clopidogrel (figura 3). La dosis de $100 \mathrm{mg} /$ día de aspirina fue la prescrita con más frecuencia (78.6\%), seguida de $200 \mathrm{mg} /$ día y $300 \mathrm{mg} /$ día en $4.8 \%$ y $14.3 \%$, respectivamente.

Entre las estatinas, la lovastatina se indicó a 39 pacientes con dosis de $20 \mathrm{mg} /$ día y a 6 pacientes con 40 $\mathrm{mg} /$ día; y solo se registró un paciente con atorvastatina a dosis de $20 \mathrm{mg} /$ día.

En cuanto a la prescripción de los beta-bloqueadores, 17 pacientes estaban con atenolol $25 \mathrm{mg} /$ día, 20 con 50 $\mathrm{mg}$ /día y otro más con $100 \mathrm{mg}$ /día. Solamente a una persona se le prescribió carvedilol $50 \mathrm{mg} /$ día.

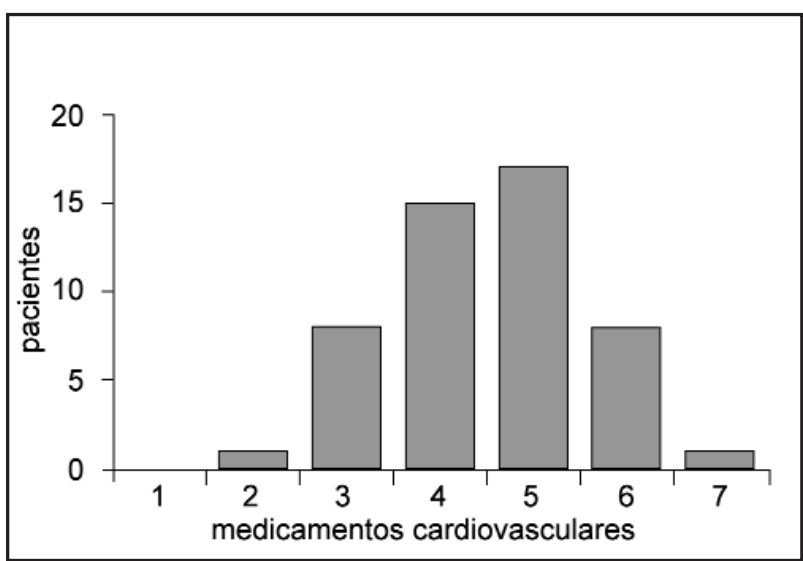

Figura 1. Distribución de los 50 pacientes con un primer IAM egresados vivos según el número de medicamentos prescritos para prevención secundaria al egreso.

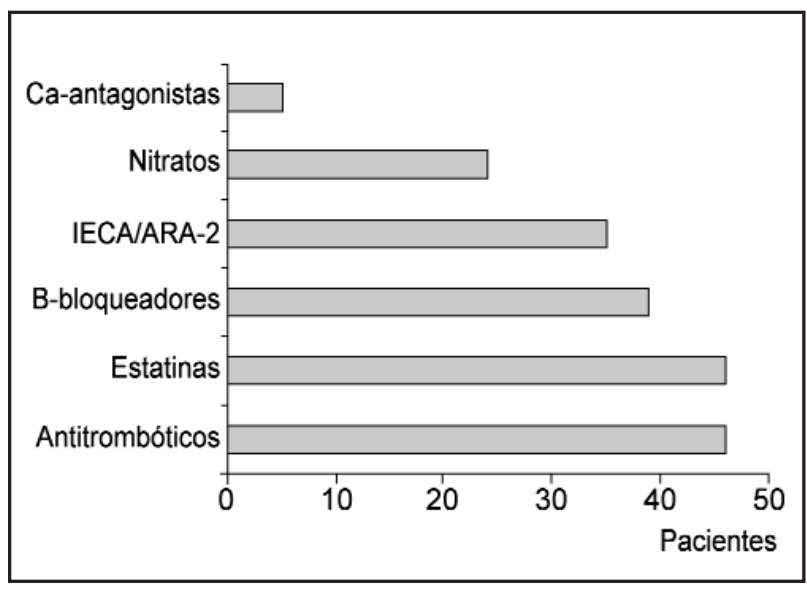

Figura 2. Medicamentos cardiovasculares prescritos para prevención secundaria en 50 pacientes con un primer IAM egresados vivos. 


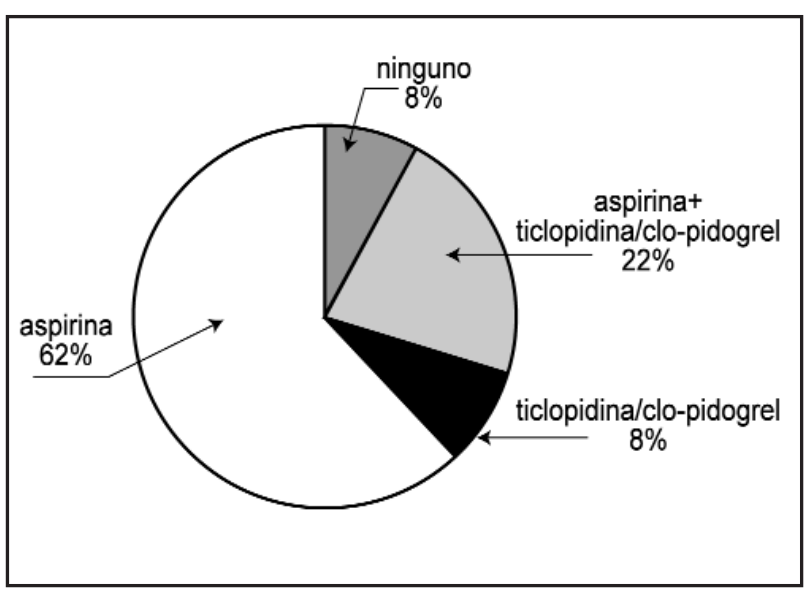

Figura 3. Distribución de 50 pacientes con un primer IAM egresados vivos según la prescripción de antitrombóticos.

Al 70\% de los pacientes se les prescribió enalapril, la dosis predominante fue de $5 \mathrm{mg}$ /día para 22 de ellos (62.9\%), mientras que a 4 pacientes se les prescribió 2.5 $\mathrm{mg} / \mathrm{día}$, a 2 pacientes $10 \mathrm{mg} / \mathrm{d}$, a 6 personas $20 \mathrm{mg} /$ día y una más tenía prescrito enalapril $40 \mathrm{mg}$ /día

Se documentó la prescripción de vasodilatadores directos a 20 pacientes $(40 \%), 8$ con dinitrato de isosorbide (dosis $10-60 \mathrm{mg} /$ día), 8 con nitroglicerina para uso sublingual y 4 con ambos.

Otros medicamentos prescritos fueron los calcioantagonistas: amlodipina (5 pacientes), un antiarrítmico: amiodarona (3 pacientes) y un antagonista de receptor de Angiotensina 2 (ARA-2): losartán (1 paciente).

\section{Discusión}

El presente estudio se realizó con el fin de explorar la presencia de algunos factores de riesgo coronario, con énfasis en aquellos que son modificables (sobrepeso, tabaquismo, sedentarismo, etc); y de describir el perfil de la prescripción de los fármacos utilizados para la prevención secundaria del IAM al momento del egreso hospitalario.

El registro sistemático de las diversas variables de interés permitió documentar un subregistro en cuanto a la información sobre los pacientes, sus características y sus hábitos, en los expedientes clínicos; en especial cuando se trata de antecedentes relevantes para contribuir con la individualización del riesgo coronario. En este estudio se logró establecer el IMC en tan solo el $24 \%$ de los pacientes y el perfil lipídico en un $48 \%$, aún así los datos disponibles sugieren que un IAM ocurre aunque factores como el sobrepeso (IMC hasta $29.9 \mathrm{~kg} / \mathrm{m}^{2}$ ) ${ }^{8}$ o bien la misma hipercolesterolemia no parecen alcanzar valores notablemente elevados.
En particular, los niveles séricos de colesterol total y del LDL-colesterol son considerados como factores de riesgo mayores para la aparición de eventos recurrentes en pacientes con IAM, por lo cual se ha recomendado proceder con su cuantificación sistemática en el curso de las primeras 24 horas post-IAM; ahí ciertamente el aspecto temporal es importante, porque los valores tienden a disminuir en el curso de las 6 semanas siguientes '. Se documentó subregistro de los niveles de colesterol y sus fracciones, lo que podría sugerir que durante la hospitalización pareció restarse interés a estas variables. Sin embargo parece evidente que la consideración al respecto de su relevancia clínica como factor de riesgo es manifiesto, dada la alta prescripción de estatinas como parte de las medidas farmacológicas para prevención secundaria. La baja frecuencia de su cuantificación se podría atribuir, al menos en parte, a que el control sistemático del perfil lipídico está incorporado dentro del marco de atención integral a la salud de las personas en el primer nivel de atención en la Seguridad Social.

En este estudio los niveles reportados de colesterol y sus fracciones deben ser interpretados con cuidado debido a que es imposible descartar la posibilidad de que un número indeterminado de pacientes estuviera tomando estatinas, por ejemplo, y así se registrarían valores menos elevados de lípidos sanguíneos, precisamente por la influencia farmacológica previa. Dada la relevancia que tiene el tema de la dislipidemia como factor de riesgo para IAM, el hecho de que para este estudio no se procuró documentar el antecedente de medicación específica para la hipercolesterolemia, a posteriori se considera una limitación derivada del diseño y sus variables de interés.

En el contexto de los riesgos, se pone en evidencia que se mantiene la contribución de la hipertensión y la diabetes como factores constantemente asociados al evento coronario, los cuales ameritan un control médico estricto y la implementación permanente de medidas no farmacológicas ${ }^{1,8,9}$.

La proporción de uso de los antitrombóticos, encabezados específicamente por la aspirina, de los beta-bloqueadores, principalmente atenolol, y de los IECAs, en particular enalapril, pone de manifiesto una discreta subutilización en evidente discordancia con las recomendaciones para intervención farmacológica en procura de la prevención secundaria, pues estos medicamentos se recomiendan como de uso rutinario para todos los pacientes con IAM, salvo contraindicación ${ }^{1-3}$.

Al considerar que la aspirina debe ser administrada rutinariamente y continuada por tiempo indefinido durante la vida de los pacientes con enfermedad coronaria y que la dosis oral recomendada era de $75-150 \mathrm{mg} /$ día $^{1,9}$, con este estudio se demostró una tendencia a la infraprescripción, pues apenas al $92 \%$ de los pacientes les fueron prescritos los antitrombóticos al egreso; y, tal como se esperaba, predominó el uso de la aspirina y de la dosis de $100 \mathrm{mg} /$ día. En esta serie de pacientes con un IAM, la necesidad de prescribir un antitrombótico alternativo a la aspirina alcanzó un $8 \%$, cifra que puede ser considerada como un indicador preliminar del requerimiento local. 
Por otra parte, dado que para el presente estudio piloto se contó con una muestra de 50 personas, es necesario considerar que el tamaño actual de la misma ciertamente podría constituir un factor limitante para la generalización de los hallazgos a las características de la población portadora de enfermedad coronaria que sobreviven a un primer evento; no obstante, los resultados del presente informe sustentan su validación en tanto que los factores de riesgo tienden a ser coincidentes con los descritos en la literatura científica; y dada la naturaleza descriptiva del presente estudio, su relevancia surge de la proyección analítica como indicador potencial respecto a la prescripción de los medicamentos disponibles para prevención secundaria.

Finalmente, es permisible concluir que los resultados obtenidos pueden ser considerados como indicadores preliminares y sugieren que en nuestro medio, la enfermedad coronaria se tiende a manifiestar desde la etapa de adulto joven, persiste en su mayor afectación a los varones y prevalecen los factores de riesgo coronario modificables, por lo que es necesario fortalecer el ámbito preventivo en procura de su minimización. Además, como se esperaba ante un primer IAM, los antitrombóticos, los beta-bloqueadores, las estatinas, son los más frecuentemente prescritos como agentes farmacológicos para la prevención secundaria de los eventos coronarios, aunque lo óptimo es que de forma sistemática, todos los pacientes coronarios se beneficien de un abordaje farmacológico con toda la medicación disponible.

\section{Agradecimiento}

Al Dr. Albin Chaves por su colaboración al contactarnos con el grupo Drug Utilization Research Group of LatinAmerica (DURG-LA), coordinador del protocolo para el estudio multicéntrico. También, al Dr. Y. Chen y a la Dra. M. Blanco, por su colaboración al recoger la información de los expedientes clínicos.

\section{Aclaración}

Datos parciales de este estudio (37 casos) se presentaron en la Sesión Anual 2003 del Grupo Latinoamericano para Estudios de Utilización de Medicamentos (DURG-LA) en Lima, Perú, el 24 de setiembre de 2003.

\section{Abstract}

Objective: To describe some factors of coronary risk present in patients who survive a first acute myocardial infartion and the pharmacological treatment prescribed for secondary prevention.
Methods: Observational, cross sectional and descriptive pilot study, information was obtained from the charts of 50 patients seen at 3 national hospitals.

Results: 42 males and 8 women, age $63 \pm 15$ years, weight $67 \pm 12 \mathrm{~kg}$, Body Mass Index $=26.1 \pm 2.1 \mathrm{~kg} / \mathrm{m} 2,38 \%$ hypertensive, $22 \%$ diabetic $(\mathrm{n}=8$ smokers patients with diabetes+hypertension) and 34\% smokers. Total cholesterol levels: $191 \pm 45.7 \mathrm{mg} / \mathrm{dL}$, cholesterol-LDL $=112 \pm 33.8$ $\mathrm{mg} / \mathrm{dL}$ and cholesterol-HDL: $39.4 \pm 8.26 \mathrm{mg} / \mathrm{dL}$. The patients were prescribed: $92 \%$ antithrombotics drugs (predominated aspirin), 92\% statins, $78 \%$ beta-blockers (atenolol or carvedilol), 70\% enalapril or losartan and 48\% nitrates.

Conclusions: this preliminary study indicateds that coronary disease appears in young adult, it's most frequent in males and showeds modifiable factors of risks. Antithrombotics drugs, beta-blockers and statins are most frequently.

\section{Referencias}

1. Hutton I (Ed.): Secondary prevention of coronary heart disease following myocardial infarction. Scottish Intercollegiate Guidelines Network. SIGN publication $N^{\circ}$ 41. Edinburgh: Royal College of Physicians, 2000.

2. Collier J (Ed.): Tratamiento del infarto agudo del miocardio. Drug Ther Bull 2000, 38: 13-16 (ed española).

3. Mehta RH, Eagle KA. Secondary prevention in acute myocardial infarction. BMJ 1998, 316: 838-42.

4. Antithrombotic Trialists' Collaboration. Collaborative meta-analysis of randomised trials of antiplatelet therapy for prevention of death, myocardial infarction and stroke in high risk patients. BMJ 2002; 324: 71-86.

5. Freemantle N, Cleland J, Young P, Mason J, Harrison J: Beta blockade after myocardial infarction: systematic review and meta-regression analysis. BMJ 1999, 318: 1730-7.

6. Yusuf S, Sleight P, Pogue J, Bosch J, Davies R, Dagenais G.: Effects of an angiotensin-converting-enzime inhibitor, ramipril, on cardiovascular events in high -risk patients. The Heart Outcomes Prevention Evaluation Study Investigators. N Engl J Med 2000, 342: 145-53.

7. Heart protection study collaborative group. MCR/BHF Heart Protection Study of cholesterol lowering with simvastatin in 20,536 high- risk individuals: a randomised placebo-controlled trial. Lancet 2002, 360: 7-22.

8. Lacy Ch.F., Armstrong L.L., Goldman M.P., Lance L.L.: Obesity treatment guidelines for adults. Summary of clinical practice guidelines National Heart, Lung and Blood Institute. In: Drug Information Handbook, 11th edition. Ontario: AphA, Lexi-Comp Inc, 2003.

9. Smith SC, Blair SN, Bonow RO, Brass LM, Cequeira MD, Dracup K, et al.: AHA/ACC guidelines for preventing heart attack and death in patients with atherosclerotic cardiovascular disease: 2000 update. J Am Coll Cardiol 2001; 38: 1581-3 